\title{
Cluster observations of a field aligned current at the dawn flank of a bursty bulk flow
}

\author{
K. Snekvik ${ }^{1}$, S. Haaland ${ }^{1,2}$, N. Østgaard ${ }^{1}$, H. Hasegawa ${ }^{3}$, R. Nakamura ${ }^{4}$, T. Takada ${ }^{3,4}$, L. Juusola ${ }^{5}$, O. Amm ${ }^{5}$, \\ F. Pitout ${ }^{2}$, H. Rème ${ }^{6}$, B. Klecker ${ }^{2}$, and E. A. Lucek ${ }^{7}$ \\ ${ }^{1}$ Department of Physics and Technology, University of Bergen, Bergen, Norway \\ ${ }^{2}$ Max-Planck-Institut für Extraterrestrische Physik, Garching, Germany \\ ${ }^{3}$ Institute of Space and Astronautical Science, Japan Aerospace Exploration Agency, Sagamihara, Japan \\ ${ }^{4}$ Space Research Institute, Austrian Academy of Sciences, Graz, Austria \\ ${ }^{5}$ Finnish Meteorological Institute, Space Research Unit, Finland \\ ${ }^{6}$ Centre d'Etude Spatiale des Rayonnements, Toulouse, France \\ ${ }^{7}$ Space and Atmospheric Physics, Imperial College, London, UK
}

Received: 3 November 2006 - Revised: 19 May 2007 - Accepted: 11 June 2007 - Published: 29 June 2007

\begin{abstract}
This article describes observations of a bursty bulk flow (BBF) in the outer central plasma sheet. The observations are made with the Cluster satellites, located approximately $19 R_{E}$ downtail, close to the midnight sector in the Southern Hemisphere. 40-60 s after Cluster first detected the $\mathrm{BBF}$, there was a large bipolar perturbation in the magnetic field. A Grad-Shafranov reconstruction has revealed that this is created by a field-aligned current at the flank of the BBF. Further analysis of the plasma moments has shown that the BBF has the properties of a depleted flux tube. Depleted flux tubes are an important theoretical model for how plasma and magnetic flux can be transported Earthward in the magnetotail as part of the Dungey cycle. The field aligned current is directed Earthward and is located at the dawn side of the BBF. Thus, it is consistent with the magnetic shear at the flank of an Earthward moving BBF. The total current has been estimated to be about 0.1 MA.
\end{abstract}

Keywords. Magnetospheric physics (Current systems; Magnetotail; Plasma convection)

\section{Introduction}

Fast flows in the magnetotail have been subject to detailed research. They are observed all the way from the tenuous plasma sheet boundary layer (PSBL) to the dense inner central plasma sheet (CPS) (Baumjohann et al., 1990). Flows in the PSBL can usually be characterized as field aligned beams (FAB) (Nakamura et al., 1992). They have distinctively different observational characteristics than flows in the middle

Correspondence to: K. Snekvik

(snekvik@ift.uib.no) of the plasma sheet. FABs typically consist of unidirectional or counterstreaming ion beams. Close to the lobes they are often Earthward directed with a low energy cutoff. Away from the lobes a counterstreaming beam can be present and the energy cutoff is lower. Further into the plasma sheet, the two beams merge to become a stagnant hot population (Nakamura et al., 1992).

Flows in the CPS are typically convective and consist of a single bulk flow (Raj et al., 2002). They are very often referred to as bursty bulk flows (BBF) (Angelopoulos et al., 1992). The name reflect that these flows are bursty by nature with velocity peaks lasting about $10 \mathrm{~s}$ (Baumjohann et al., 1990). BBFs are often accompanied by dipolarization and ion heating (Fairfield et al., 1999). Originally, the term BBFs referred to high speed flows $(>400 \mathrm{~km} / \mathrm{s})$ where plasma beta exceeds 0.5 (Angelopoulos et al., 1992). Based on these criteria, Angelopoulos et al. (1994) concluded that BBFs contribute significantly to Earthward transport of mass and flux. Plasma beta, $\beta$, is the ratio between the thermal pressure and the magnetic pressure, $B^{2} /\left(2 \mu_{0}\right)$. It is used as an indicator of where the satellite is relative to the centre of the plasma sheet.

Since FABs occasionally are observed when $\beta$ even exceeds 0.5 (e.g., Raj et al., 2002), the definition of BBFs also includes FABs. By classifying fast flows based on their ion phase space distributions, Raj et al. (2002) found that most ion distributions of fast flows fall into two distinct categories: bulk flows and field-aligned beams. Bulk flows were single drifting populations with no low energy cutoff, while field aligned beams are characterized by crescent shapes and low energy cutoffs. How field aligned the bulk flows are, depend mainly on the elevation angle of the magnetic field. 


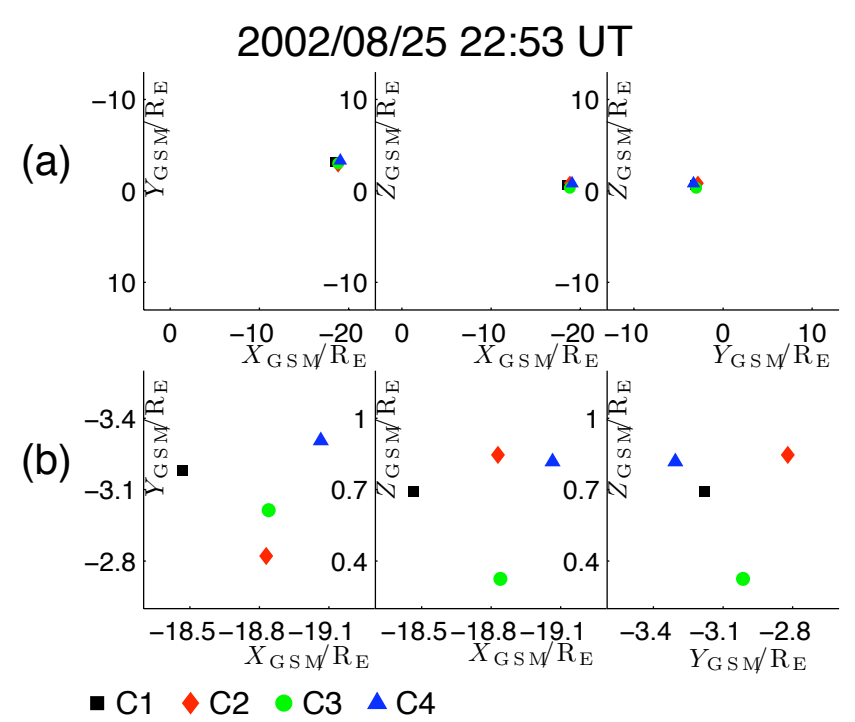

Fig. 1. (a) Position of the Cluster satellites given in GSM coordinates. (b) A magnified version of (a) showing the spacecraft tetrahedron configuration.

One theoretical explanation of BBFs is that they are depleted flux tubes (Pontius Jr. and Wolf, 1990; Chen and Wolf, 1993). When the depleted flux tubes are formed, they have lower plasma pressure than the surrounding plasma. This reduces the diamagnetic current inside the bubble. Current continuity is preserved by field aligned currents (FACs) at the flanks of the flux tube. One current is directed down into the ionosphere at the dawn side, and one current is directed up from the ionosphere at the dusk side. In a recent simulation Birn et al. (2004) predicted that the pressure balance between the depleted flux tube and the surrounding plasma will be quickly restored. In high beta regions like the CPS, this is mainly achieved by increased plasma pressure, while in low beta regions like the PSBL, the strengthening of the magnetic field is more significant. As a consequence, the plasma pressure is no longer constant along the field line and field aligned Earthward flows will take place out of the CPS into the PSBL. This is visualized in Fig. 19 of Birn et al. (2004). The initial plasma pressure reduction can be caused by a decrease in either temperature or density. In density depleted flux tubes the temperature increases to reestablish the pressure balance. At the flanks of the flux tube, the magnetic field will be twisted by flow vortices (Birn et al., 2004). This is equivalent with field aligned currents up and down at the dawn and dusk side of the BBF.

Observations of depleted flux tubes by e.g., Sergeev et al. (1996) and Nakamura et al. (2005), have confirmed the decrease in density combined with enhancement of the magnetic field. These studies also found that the boundaries of the flux tubes were tangential discontinuities. In front of the boundary there were flow shear and the magnetic field was twisted. Many boundary normals had large GSM $Y$ and $Z$ components, indicating that the spacecraft often encountered the structure at its flank.

In this paper we present and discuss Cluster data from a passage through an Earthward moving BBF with signatures of a FAC at its flank. We also estimate the dimensions and current density of the FAC region. The paper is organized as follows: in Sect. 2 an overview of the event will be given. In Sect. 3 the boundary between the BBF and the surrounding plasma will be studied, using two complementary techniques. First, we create a topological 2-D map of the structure using Grad-Shafranov reconstruction. Thereafter, we crosscheck the result with an estimation of the current based on the curlometer method and classical discontinuity analysis. We then focus on the time interval immediately after the FAC observation, and we interpret the plasma moments as consistent with the concept of BBFs as depleted flux tubes. Finally, Sect. 4 discusses the physical implications of the results, and Sect. 5 adds some conclusive remarks to the present study.

\section{Event overview}

The data presented in this paper are from the fluxgate magnetometer experiment (FGM - all spacecraft) (Balogh et al., 2001) and the Cluster Ion Spectrometry (CIS - three spacecraft) (Rème et al., 2001). CIS consists of two detectors, namely the Hot Ion Analyser (HIA), and the time-offlight ion Composition Distribution Function (CODIF). The plasma measurements used here are derived from proton data from CODIF at $\mathrm{C} 1$ and $\mathrm{C} 4$ and ion data from HIA at $\mathrm{C} 3$.

Figure 1 shows the Cluster position and the tetrahedron configuration on 25 August 2002 22:53 UT in GSM coordinates. Cluster was located around $19 R_{E}$ downtail in the post midnight sector during the event studied here. The spacecraft configuration forms an almost regular sided tetrahedron (Robert et al., 1998b) with separation distances between $2300 \mathrm{~km}$ and $2900 \mathrm{~km}$. C1 is the most Earthward satellite while C4 is most tailward. C2 is most duskward and C4 is most dawnward. The most southward satellite is C3.

Figure 2 shows an overview of some Cluster observations during the interval 22:50 UT-23:00 UT. Plasma beta is used as a proxy of the distance from the neutral sheet. Only the $\mathrm{X}$ and $\mathrm{Y}$ components of the magnetic field are used to calculate plasma beta. As noted by Raj et al. (2002), bulk flows are often associated with increased $B_{Z}$. Including $B_{Z}$ in plasma beta could therefore lead to the incorrect interpretation that the spacecraft is moving away from the neutral sheet during bulk flows. In addition, only the perpendicular pressure component is used. Plasma beta derived this way is written $\beta_{X Y}$ throughout the rest of this text. A similar approach was also used by Nakamura et al. (2004).

To guide the reader we have used color codes to identify key regions; lobe (gray), PSBL (white) and CPS (yellow). 


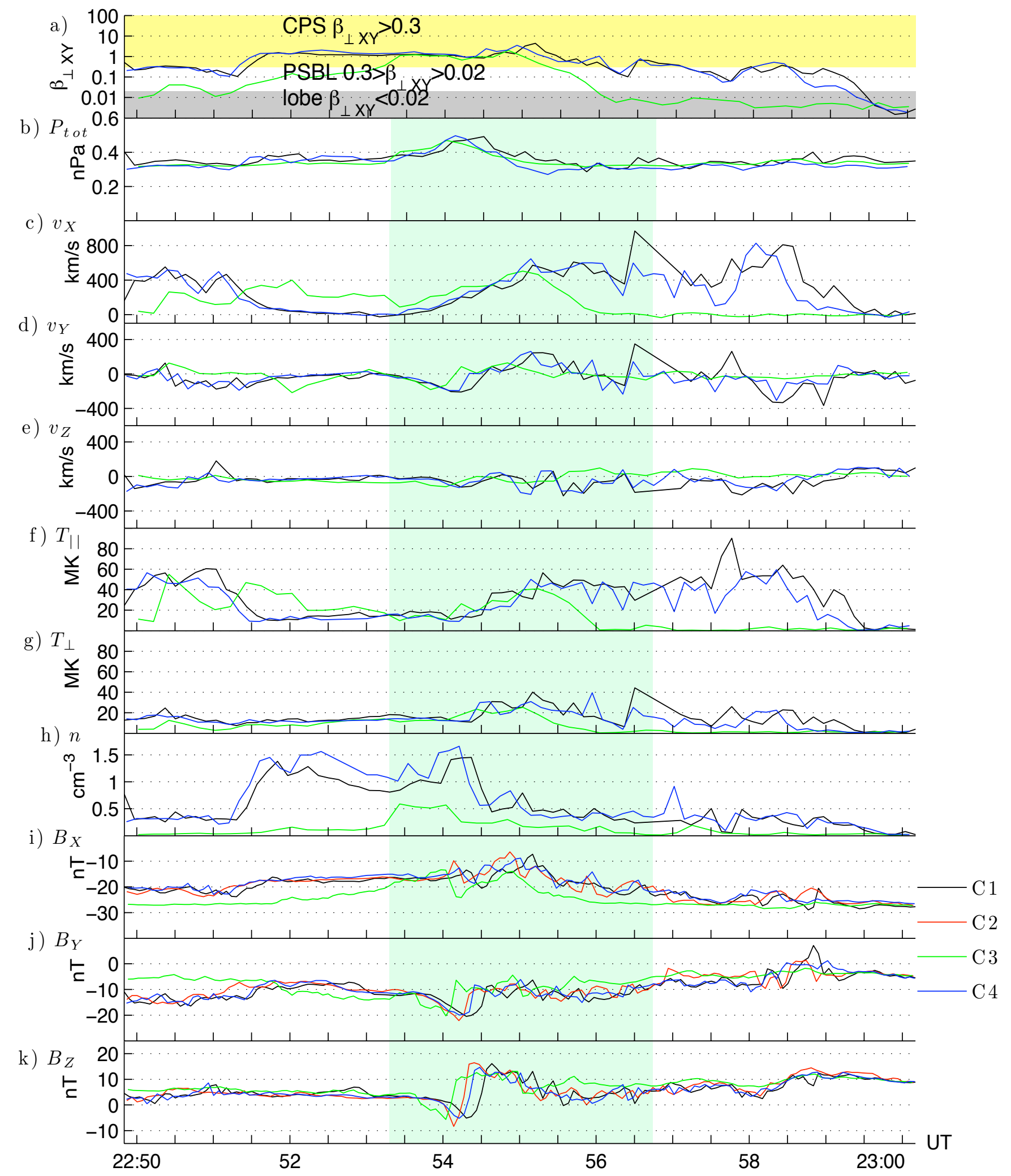

Fig. 2. Overview of key parameters during the event. The panels show: (a) Plasma beta. Background colors indicate regions of the plasma sheet based on results from Baumjohann et al. (1988) and Baumjohann et al. (1989). (b) Total pressure. (c-e) GSM components of the plasma velocity. (f-g) Parallel and perpendicular temperatures. (h) Proton density $(\mathrm{C} 1, \mathrm{C} 4)$ and ion density (C3). (i-k) GSM components of the magnetic field. The standard spacecraft color scheme is used. The green shaded interval from 22:53:17 to 22:53:44 indicate the BBF event. 
This classification is based on the beta value and the statistical results from Baumjohann et al. (1988) and Baumjohann et al. (1989). They found that typical values for plasma beta in the magnetotail are 0.02 in the outer part of the PSBL, 0.05 in the inner part of the PSBL, 0.3 in the outer CPS, 3 in the inner CPS and 30 in the neutral sheet. In this article $\beta_{X Y}$ equal to 0.02 is used as the boundary between the lobe and the PSBL and 0.3 as the boundary between the PSBL and the CPS. The total pressure (Fig. 2b), is the sum of the plasma pressure and the magnetic pressure.

During the time interval 22:50-23:00 UT, the satellites move from the CPS into the southern lobe. The negative $B_{X}$ indicate that they are located south of the neutral sheet. Notice that the $B_{Y}$-component is quite large throughout the interval. The $B_{Z}$-component is quite stable except around 22:54 UT where it becomes significantly perturbed.

The time interval 22:53:17-22:56:44 UT (shaded with green background color) shows the passage of a BBF. To identify the BBF, we have used the same definition as Angelopoulos et al. (1992) with the extension for 3 Cluster satellites introduced by Cao et al. (2006), namely a time interval where the ion flow velocity, $v$, and plasma beta exceed $100 \mathrm{~km} / \mathrm{s}$ and 0.5 , respectively, and contain at least one sample with $v>400 \mathrm{~km} / \mathrm{s}$. Subsequent segments of $v>400 \mathrm{~km} / \mathrm{s}$ within a 10 min interval are regarded as a single BBF even if the velocity and plasma beta values between them drop to below $100 \mathrm{~km} / \mathrm{s}$ and 0.5 , respectively.

In the beginning of the BBF interval $(\sim 22: 53 \mathrm{UT})$, all four spacecraft are in the outer CPS where $\beta_{X Y} \approx 1$ and $\left|B_{X}\right| \approx 18 \mathrm{nT}$. The velocity increases gradually to about $600 \mathrm{~km} / \mathrm{s}$ around 22:55 UT. The flow is mainly Earthward. After 22:55 UT all spacecraft observe a decrease in plasma beta combined with an increase in $B_{X}$, indicating a movement of the spacecraft towards the lobe. $\mathrm{C} 3$ exits the plasma sheet short after, while $\mathrm{C} 1$ and $\mathrm{C} 4$ move in and out of the CPS a few times near the end of the BBF time interval.

\section{Field aligned currents at the BBF boundary}

The BBF interval marked with green background color in Fig. 2 starts 22:53:17 with a gradual increase in the velocity and the total pressure combined with dawnward and lobeward rotation of the magnetic field. This lasts until 22:54:0022:54:20 UT (depending on the spacecraft in consideration) and ends with a large perturbation in $B_{Y}$ and $B_{Z}$. In this section we take a closer look at this $B$-field signature, and argue that it is the results of a region of FAC along the boundary/flank of the BBF.

\subsection{Reconstruction of the boundary of the BBF}

Grad-Shafranov (GS) reconstruction (Hau and Sonnerup, 1999) is a technique for identifying the topology of structures in the magnetic field based on in situ measurements. It was originally a single spacecraft technique, but has recently been improved to utilize measurements from several spacecraft (Hasegawa et al., 2005). GS reconstruction provides a map of the magnetic field and the plasma in a region around the spacecraft trajectory. The technique is based on some assumptions about the structure (see Hau and Sonnerup, 1999, for a detailed description):

- There must exist a frame where the structure is approximately time stationary $\left(\frac{\partial}{\partial t} \cong 0\right.$ ). This frame is co-moving with the structure. In the following, quantities in this frame will be referred to with a prime.

- The spatial derivative in one direction must be much smaller than in the two other directions, i.e. the structure must be approximately 2 -dimensional. The invariant direction is taken as the $Z^{\prime}$-axis, i.e. $\frac{\partial}{\partial Z^{\prime}} \cong 0$.

- The pressure must be isotropic.

- The inertia terms in the momentum equation can be neglected.

The velocity of the structure is obtained from a deHoffmannTeller (HT) analysis (Khrabrov and Sonnerup, 1998). A frame where $\boldsymbol{E}=0$, will be time stationary because $\frac{\partial \boldsymbol{B}}{\partial t}=-\nabla \times \boldsymbol{E}=\mathbf{0}$. In the real world there is no frame where the electric field vanishes, but it is still possible to find the velocity, $\boldsymbol{V}_{\mathrm{HT}}$, where the electric field is as small as possible. In our case, $-\boldsymbol{v} \times \boldsymbol{B}$ has been used as a proxy of $\boldsymbol{E}$. Using data from $\mathrm{C} 1$ and $\mathrm{C} 3$ for the time interval 22:53:3022:55:15 UT we found the deHoffmann-Teller frame to be $\boldsymbol{V}_{\mathrm{HT}}=[472,155,-166] \mathrm{km} / \mathrm{s}(\mathrm{GSM})$.

Based on the assumptions above, the GS equation for the partial vector potential $A\left(X^{\prime}, Y^{\prime}\right)$, can be derived:

$\frac{\partial^{2} A}{\partial X^{\prime 2}}+\frac{\partial^{2} A}{\partial Y^{\prime 2}}=-\mu_{0} \frac{\mathrm{d}\left(p+B_{Z^{\prime}}^{2} /\left(2 \mu_{0}\right)\right)}{\mathrm{d} A}=-\mu_{0} j_{Z^{\prime}}$,

where

$\boldsymbol{B}^{\prime}=\left[\frac{\partial A}{\partial Y^{\prime}},-\frac{\partial A}{\partial X^{\prime}}, B_{Z^{\prime}}\left(X^{\prime}, Y^{\prime}\right)\right]$.

By measuring pressure and magnetic field, the GS-equation can be used to make maps of magnetic field, pressure and current. The direction of the invariant $Z^{\prime}$-axis is chosen such that the correlation coefficient between the fields measured by the satellites and those from the map is optimized. The $X^{\prime}$-axis is chosen such that it is parallel with the component of $V_{\mathrm{HT}}$ perpendicular to the $Z^{\prime}$-axis. The unit vectors defining the GS reconstruction plane, $X^{\prime}, Y^{\prime}$ as well as the invariant axis $Z^{\prime}$ and $V_{H T}$ are given in Table 1. For our event, the $Y^{\prime}$-axis almost points in $Z_{\mathrm{GSM}}$ direction, whereas the invariant axis, $Z^{\prime}$, lies near the $X_{\mathrm{GSM}}-Y_{\mathrm{GSM}}$ plane.

Figure 3 shows the result of the GS reconstruction; maps of the magnetic field (upper panel) and current density (lower panel). The maps are produced by merging four magnetic 
field maps, each reconstructed from one of the four spacecraft. White arrows in the plot show the measured magnetic field (top panel) and velocity in the co-moving frame (bottom panel), respectively, along the spacecraft trajectories. For reference, we have also drawn the Cluster tetrahedron configuration and the relation to the GSM coordinate system in the upper panel. The correlation coefficient between the three magnetic field components predicted from the map at points along the four spacecraft trajectories and those actually measured, is 0.988 . This good agreement indicates that the invariant axis and reference frame are well determined, and also that the 2-D assumption is satisfactory.

The GS frame should ideally be co-moving with the BBF. In such a frame, the BBF appears to be at rest while the Cluster satellites are moving from the left to the right side in Fig. 3 with $220 \mathrm{~km} / \mathrm{s}$. The upper part of the maps, corresponding to near-CPS region, is not so reliable since we have no observations from this region. The maps represents cuts approximately perpendicular to the background magnetic field as seen from the Earth.

The most notable feature apparent in Fig. 3 is the shear of the magnetic field lines near the center of the maps. Note that the magnetic field lines are not really closed loops because of the large $B_{Z^{\prime}}$ into the plane. The result of the shear is an elongated region with intensified electric current, visualised in the bottom panel of Fig. 3. From this reconstruction one can infer that the region of enhanced current is at least 2500 by $5000 \mathrm{~km}$, with an average current density of approximately $8 \mathrm{nA} / \mathrm{m}^{2}$. The total current is then of the order of $0.1 \mathrm{MA}$. The reconstruction reveals a field aligned current (FAC), although antiparallel to the magnetic field.

The motion of the plasma in Fig. 3 can be better understood if one interprets the FAC as the boundary of the BBF. From the frame which is co-moving with the BBF, one will see plasma moving towards the BBF from the left. The BBF is an obstacle which the plasma must flow around and closer to the $\mathrm{BBF}$ the flow changes direction to move around the boundary. In an ideal MHD picture, the plasma flow carries the magnetic field with it. This interpretation will be discussed further in Sect. 4.

To verify the GS results, we have also estimated the current with the curlometer method based on all four spacecraft (Harvey, 1998; Robert et al., 1998a). The curlometer method should ideally only be used when the magnetic field is varying linearly between the Cluster satellites. This condition is not very well satisfied for our event because the $B_{Z}$ reversal has already ended for Cluster 3 when it begins for Cluster 1 in Fig. 2k. This will probably cause the current density to be underestimated because scales smaller than the distance between the spacecraft are smeared out. Still, given a regular sided tetrahedron, the curlometer method will provide the correct direction in most cases (Dunlop and Balogh, 2005).

Figure 4 shows the parallel and perpendicular components of the estimated current. Also shown is the ratio between the magnitudes of the estimated divergence and curl of the
Table 1. The coordinate axes and the velocity of the GS frame.

\begin{tabular}{ll}
\hline Description & vector $(\mathrm{GSM})$ \\
\hline deHoffmann-Teller velocity & $\boldsymbol{V}_{\mathrm{HT}}=[472,155,-166] \mathrm{km} / \mathrm{s}$ \\
GS reconstruction $X^{\prime}$-axis & {$[-0.585,0.737,0.338]$} \\
GS reconstruction $Y^{\prime}$-axis & {$[0.295,-0.195,0.936]$} \\
GS reconstruction $Z^{\prime}$-axis & {$[0.755,0.647,-0.103]$} \\
\hline
\end{tabular}

magnetic field. Since $\nabla \cdot \boldsymbol{B}=0$, this is a measure of the quality of the current estimation. When this ratio is about one or greater, the current estimate must be treated with caution.

Nevertheless, the curlometer results seem to agree well with the GS reconstruction. The curlometer method also confirms that the current is field aligned. As shown by the green line, the quality of the estimation is not so good when the estimated FAC starts and when it ends, however, there is an interval in between where the ratio is low, and the current estimate should be fairly reliable.

\subsection{Orientation and motion of the BBF boundary}

To crosscheck the orientation and dimensions provided by the GS reconstruction, we also perform a "classical" analysis of the BBF boundary. As seen in Fig. 3, the GS reconstruction reveals a structure with scale sizes similar to or smaller than the spacecraft separation distance. Four spacecraft timing analysis will therefore be subject to errors. However, as shown by e.g. Sonnerup et al. (2006); Haaland et al. (2004), single spacecraft methods can provide very accurate estimations of boundary orientations if the underlying physical model assumptions are valid.

The orientation of a boundary is typically described by its normal vector, $\hat{\boldsymbol{n}}$. We assume that the boundary between the $\mathrm{BBF}$ and the surrounding plasma is a tangential discontinuity (TD), similar to what has been observed by others (e.g. Sergeev et al., 1996). The TD normals lie in the direction in which the magnetic field is smallest in a least square sense during the interval the satellite crosses the boundary. This means minimizing $\left\langle(\boldsymbol{B} \cdot \hat{\boldsymbol{n}})^{2}\right\rangle$ with respect to $\hat{\boldsymbol{n}}$, i.e. obtaining the rank-2 least square approximation of the magnetic field. The method was originally developed by Siscoe et al. (1968). This procedure is performed for each spacecraft separately, giving four different TD normals from different positions of the BBF boundary. The time intervals used to establish the orientation will include the sharp increase in $B_{Z}$ (Fig. 2k) with an additional $5 \mathrm{~s}$ before and after the minimum and maximum. The estimated TD normals from each spacecraft, the time intervals used for the analysis, and the average normal magnetic field component, $\left\langle B_{n}\right\rangle=\langle\boldsymbol{B} \cdot \hat{\boldsymbol{n}}\rangle$, along with its standard deviation, $\sigma$, are shown in Table 2. Both $\left\langle B_{n}\right\rangle$ and $\sigma$ are small for all four spacecraft, compared with the average magnetic field. The TD normals differ most between C2 and C3. 

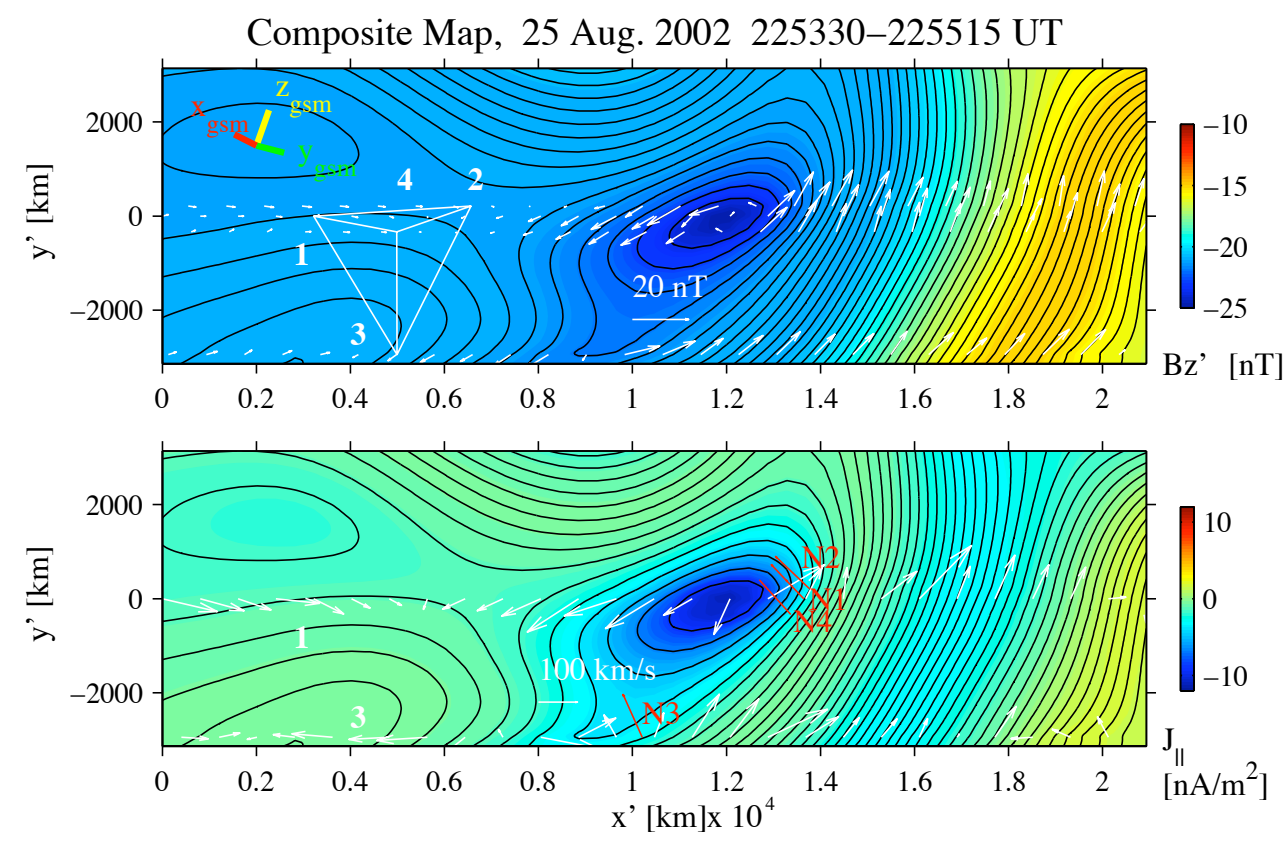

Fig. 3. Grad Shafranov reconstruction of the boundary of the BBF. Top panel: Map of the magnetic field. Black lines show magnetic line contours in the reconstruction plane and the color indicates the out-of-plane component of the $B$-field (negative into the plane). White arrows show the measured magnetic field along the trajectories of the four spacecraft. Bottom panel: Similar to top panel, but the color now indicates the out-of-plane current density (positive into the plane) and the white arrows indicate measured flow velocities, relative to the GS frame. Red arrows show boundary normals obtained as described in Sect. 3.2. The center of the maps reveal an about $2500 \times 5000 \mathrm{~km}$ structure with a helical like magnetic field topology and a strong field aligned current component. The coordinate system in this figure is not GSM, but uses optimally selected axes for the reconstruction. The orientation of the GSM coordinates is indicated in the upper panel and also listed in Table 1 .

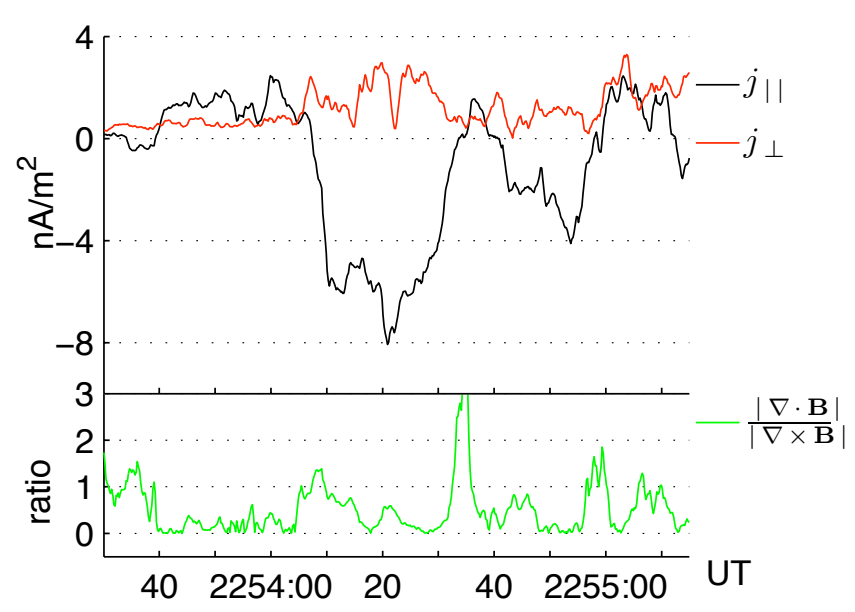

Fig. 4. Current density from the curlometer method. Top panel: $j_{\|}$(black line) and $j_{\perp}$ (red line). Bottom panel: The ratio $|\nabla \cdot \boldsymbol{B}| /|\nabla \times \boldsymbol{B}|$, which is an indicator of the quality of the current estimation. The interval shown is the same as the interval used for the GS reconstruction. A strong field aligned current is observed in the interval 22:54:05-22:54:35 UT.
The angle between the two normals is $28^{\circ}$. This indicates that the BBF is moderately large compared to the Cluster tetrahedron.

The last row of Table 2 show the estimated thickness of the boundary, estimated from the equation $\left(\boldsymbol{V}_{\mathrm{HT}} \cdot \hat{\boldsymbol{n}}\right) \cdot \Delta t$, where $\Delta t$ is the time for the satellite to cross the boundary. Each crossing was defined as an uninterrupted interval where the increase of $B_{Z}$ exceeds $0.2 \mathrm{nT} / \mathrm{s}$. This specific value was found by visual inspection. The estimated thicknesses for $\mathrm{C} 1, \mathrm{C} 2$ and $\mathrm{C} 4$ range from $2100-2300 \mathrm{~km}$. This is also consistent with the $2500 \mathrm{~km}$ width obtained from the GS reconstruction. For C3, the inferred normal is almost perpendicular to $\boldsymbol{V}_{\mathrm{HT}}$, and no reliable thickness can be derived.

Based on the TD normals estimated for each spacecraft, the time each spacecraft spends crossing the boundary and the deHoffmann-Teller velocity, it is possible to estimate the two principal curvatures of a plasma structure (Mottez and Chanteur, 1994). In our case they have been estimated to be $k p_{1}=7 R_{E}$ and $k p_{2}=2 R_{E}$. The two curvatures lie in orthogonal planes where each plane is defined by the average TD normal and the tangent vectors to each curvature. The tangent vectors are $[0.637,0.769,0.060]$ and $[-0.421,0.281$, 0.863 ] in GSM for $k p_{1}$ and $k p_{2}$, respectively. The largest curvature radius, $k p_{1}$, is mainly in the $X_{\mathrm{GSM}}-Y_{\mathrm{GSM}}$ plane 
Table 2. The tangential discontinuity normals of the boundary and the time intervals used to find them, for each of the spacecraft. In the third row the average magnetic field components along the normals and their standard deviations are given. The fourth row shows the estimated thickness of the boundary.

\begin{tabular}{|c|c|c|c|c|}
\hline & $\mathrm{C} 1$ & $\mathrm{C} 2$ & $\mathrm{C} 3$ & $\mathrm{C} 4$ \\
\hline TD normal (GSM) & {$\left[\begin{array}{lll}0.660 & -0.612 & 0.437\end{array}\right]$} & {$\left[\begin{array}{lll}0.731 & -0.568 & 0.379\end{array}\right]$} & {$\left[\begin{array}{lll}0.473 & -0.507 & 0.720\end{array}\right]$} & {$[0.677,-0.579,0.455]$} \\
\hline Time interval (UT) & 22:54:12-22:54:41 & 22:54:02-22:54:27 & 22:53:55-22:54:17 & 22:54:09-22:54:30 \\
\hline$\langle\boldsymbol{B} \cdot \hat{\boldsymbol{n}}\rangle_{-}^{+} \sigma[\mathrm{nTT}]$ & $-0.1_{-}^{+} 1.7$ & $0.0_{-}^{+} 1.6$ & $0.1_{-}^{+} 1.0$ & $0.1_{-}^{+} 1.6$ \\
\hline Thickness [km] & 2300 & 2300 & - & 2100 \\
\hline
\end{tabular}

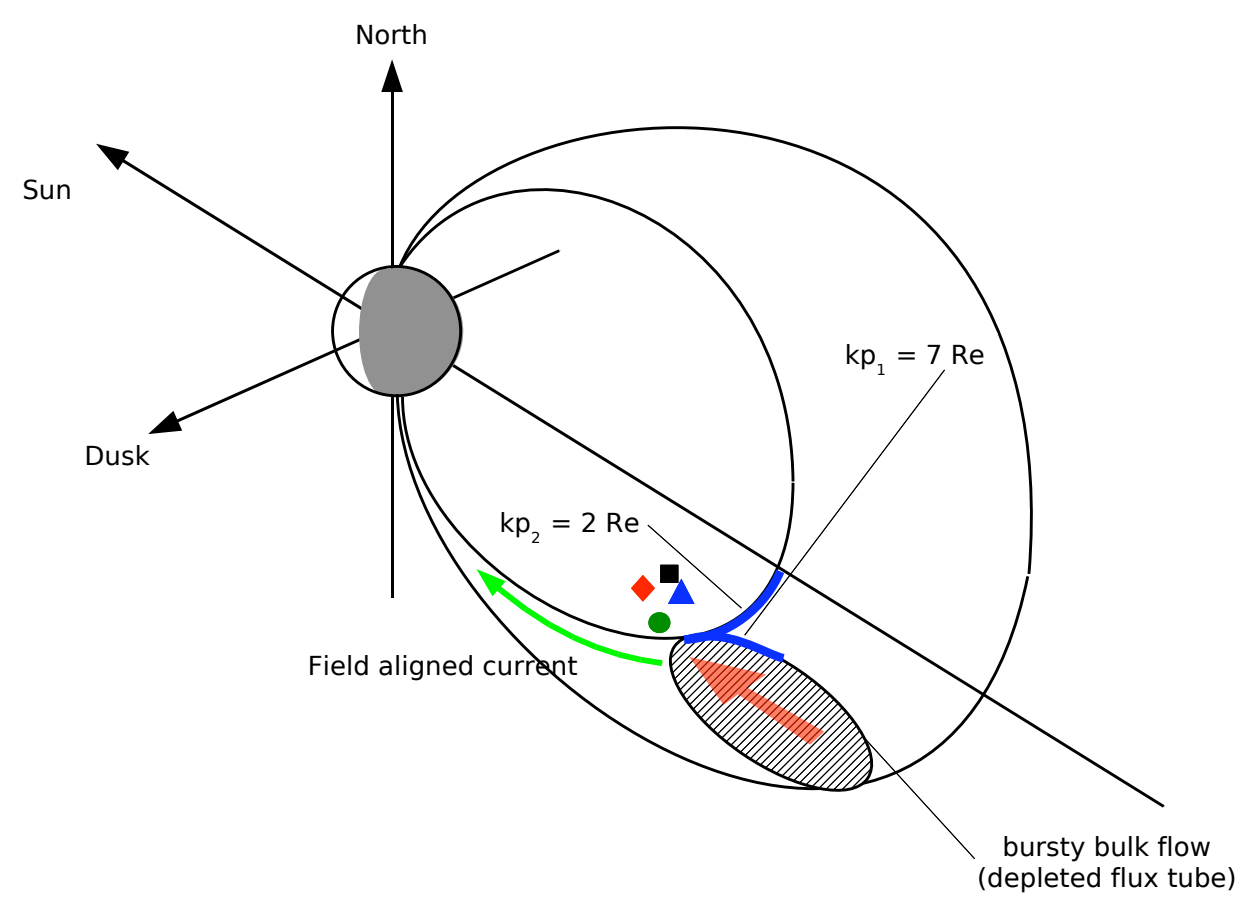

Fig. 5. A schematic overview of the orientation of the BBF. The Cluster quartet, located below the neutral sheet, encounters the dawn flank of the BBF, and observes a downward (into the ionosphere) field aligned current. The magnitude of the local curvatures kp1 and kp2 (indicated by the blue curve segments), of the structure is estimated to be 7 and $2 R_{E}$, respectively. Adapted from Birn et al. (2004).

and very close to the invariant $Z^{\prime}$-axis from the GS reconstruction, while $k p_{2}$ lies in the $X^{\prime}-Y^{\prime}$ plane. Also interesting is that the curvature radius of the flux tube can be estimated from the right part of the map (Fig. 3) to be $1-2 R_{E}$. The results from the independent analyses are mutually consistent.

Figure 5 shows a schematic interpretation of some of the results from this section. Based on both the direction of the boundary normals and the principal curvatures, it seems like the Cluster satellites encounter the boundary of a BBF, somewhere between its front and dawnward flank below the neutral sheet. The blue lines show the estimated curvatures where the satellites encounter the boundary of the BBF. $k p_{1}$ has been interpreted as the local radius of curvature of a cross section of a flux tube. The width of the flux tube is not known, but BBFs are reported to have an average width of 2-3 $R_{E}$ (Nakamura et al., 2004). If this is valid for this event, it suggests an elongated structure as outlined in Fig. 5. The smallest curvature radius, $k p_{2}$, represent the curvature of the flux tube and can be interpreted as a proxy of the half thickness of the current sheet.

\subsection{BBFs as generator of field aligned current}

One possible mechanism for generating field aligned currents, is the motion of underpopulated flux tubes (Chen and Wolf, 1993). When such flux tubes move Earthward, they push away plasma in front of them, creating flow vortices and shear in the magnetic field. The magnetic shear will generate field aligned currents (Birn et al., 2004).

Figure 6 shows some key parameters during the full BBF interval, 22:53:17-22:56:44 UT. Since all three spacecraft 


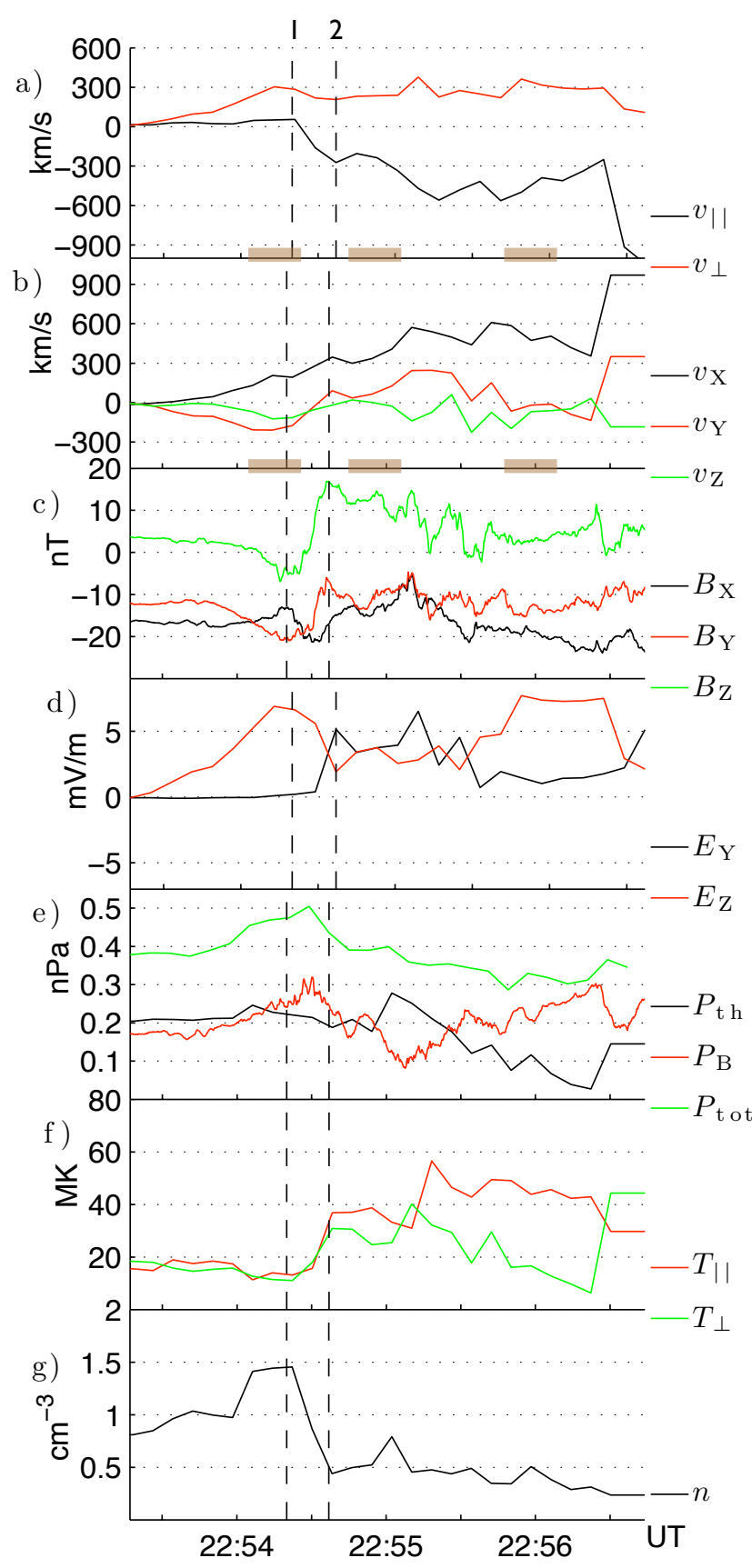

Fig. 6. Measurements from Cluster 1 during the interval 22:53:1722:56:44 UT (the interval marked with green background in Fig. 2). The panels show: (a) plasma flow parallel and perpendicular to the magnetic field; (b) GSM components of the velocity; (c) GSM components of the magnetic field; (d) $Y_{\mathrm{GSM}}$ and $Z_{\mathrm{GSM}}$ components of the convection electric field $(\boldsymbol{E}=-\boldsymbol{v} \times \boldsymbol{B})$; (e) plasma pressure, magnetic pressure and total pressure; (f) temperature components parallel and perpendicular to the magnetic field (g): ion density. Vertical lines mark the boundary of the flux tube, and brown horizontal bars indicate intervals used to generate ion distibution plots shown in Fig. 7.
SC1, SC3 and SC4 show similar characteristics, we only show data from $\mathrm{SC} 1$ in this figure.

Prior to 22:54:20 UT (vertical line 1), $v_{Y}$ and $v_{Z}$ (panel b) gradually increase (becomes more negative). The flow is mainly perpendicular to the magnetic field. Also $B_{Y}$ and $B_{Z}$ decrease. During the same interval the total pressure increases. It is mainly the magnetic pressure which contributes to this. This is interpreted as the effect of the BBF plowing through the plasma. The magnetic field is draped around the BBF and the surrounding plasma is "pushed" away. This has been reported earlier by e.g., Sergeev et al. (1996); Nakamura et al. (2005). There are also indications of compression of plasma in front of the BBF as seen in the increased density (panel g).

The time interval between the first and second line is interpreted as the boundary of the BBF. This is where we observe the large field aligned current. The perpendicular velocity decreases and the parallel velocity increases. $v_{Y}$ and $v_{Z}$ decrease to zero. Simultaneously, both $B_{Z}$ and $E_{Y}$ increases.

There are three key observations in the figure which support the interpretation of the BBF as a depleted flux tube with an initial density reduction (Birn et al., 2004): 1) A decrease in the density starting around 22:54:20 UT (panel g). 2) An increase in temperature around the same time (panel f). 3) A dipolarization of the magnetic field (panel c). In addition, the $Y$-component of the electric field is enhanced (panel d).

The interval after 22:54:35 UT (second vertical line) is interpreted as the BBF proper. The flow velocities are still high, but the density is lower than the initial density, and the temperature is higher than the initial temperature.

Figure 7 shows three snapshots of the ion distribution functions. The time interval of each distribution is marked with brown lines in Fig. 6. The intervals were carefully selected so that there are no abrupt changes of the distributions during each interval. The leftmost distribution represents the plasma surrounding the BBF. The effect of the BBF plowing through the plasma can be seen as a relatively small, but distinct perpendicular velocity. The next two distributions are typical for FAB. They have the typical crescent shape, and a counterstreaming beam is present. The second distribution is centered around $v_{\perp}=-v_{\|}=500 \mathrm{~km} / \mathrm{s}$. We interpret this as a FAB in a depleted flux tube with large convective velocity.

It is not easy to define the exact time when the spacecraft leaves the depleted flux tube. But between 22:55:00 and 22:56:00 UT, the dipolarization, the perpendicular temperature and the $Y$-component of the electric field gradually decrease (Fig. 6). Therefore, at the time of the third distribution (Fig. 7), the spacecraft has most likely left the depleted flux tube. This is supported by the typical boundary layer type distribution. Since the spacecraft leave the flux tube towards the PSBL, the density remains low. 


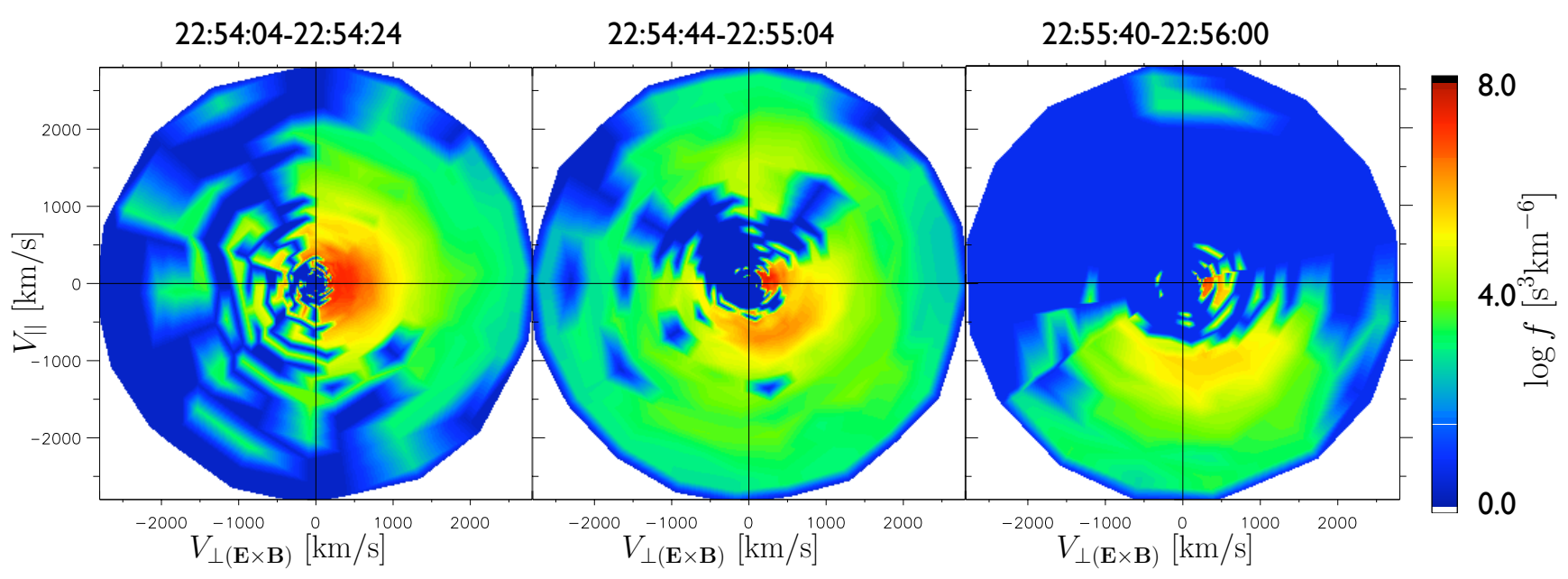

Fig. 7. Proton distributions from CODIF at Cluster 1 for the 3 time intervals shown in Fig. 6 a and 6 b by three brown lines.

\section{Discussion}

The theory that BBFs can have field aligned currents at its flanks is not new. Pontius Jr. and Wolf (1990) proposed that flow channels with lower density than the surroundings will create a localized current wedge with upward current at the duskward edge and downward current at the dawnward edge. These flow channels take the form of underpopulated flux tubes. This idea is important because it may provide a solution to the so called "pressure crisis" (Erickson and Wolf, 1980) in the magnetotail. The BBF analyzed here shows the predicted signatures (Birn et al., 2004) of a depleted flux tube. Inside the BBF there is dipolarization and enhanced $E_{Y}$, and the density is about half of what it is outside. Pressure balance between the flux tube and the surrounding plasma is maintained by higher temperature inside the flux tube. At the front of the BBF, the density is larger, indicating that the plasma is compressed.

Observationally, BBF associated FACs are characterized by abrupt perturbations in the magnetic field near the leading edge of the flow. Two different processes will contribute to the perturbation. (1) In front of the flux tube a shear flow pattern will develop, which displaces the ambient plasma so that the flux tube can move Earthward (Sergeev et al., 1996). (2) A depleted flux tube is characterized by stronger $B_{Z}$ than the surrounding flux tubes. The shear region correspond to field aligned currents. The exact nature of the magnetic field disturbance is critically dependent on where the spacecraft encounter the flux tube. In the center of the plasma sheet, where the current is out of the $X-Y$ plane, the sheared magnetic field is mainly seen in $B_{X}$ and $B_{Y}$. There will also be an increase in $B_{Z}$ inside the main flow (Sergeev et al., 1996). In the PSBL, far from the centre, a simulation by Birn et al. (2004) predicts the FAC to be at the inner edge of the flux tube giving largest shear in $B_{Y}$ in Z-direction, i.e., $\partial B_{Y} / \partial Z$. The dipolarization inside the flux tube is likely to be less prominent here. In our case the spacecraft enter the flow from the side in the outer CPS and detect negative excursion in $B_{Z}$ and $B_{Y}$ before entering the main flow with large $B_{Z}$. A gradual change of the position of the FAC (the shear region) from being at the flank in the CPS to the inner edge in the PSBL may explain this. The relatively larger Zcomponent of the $\mathrm{C} 3$ boundary normal (Table 2), compared with the other normals, is consistent with this. In the MHD model used by Birn et al. (2004), a negative $v_{Z}$ before the front (Fig. 6), can be understood as the flux tube plowing the ambient plasma southward. This flow fills the void left behind by the Earthward moving flux tube.

The $B_{Z}$ signature described in this paper has a seemingly similar signature as Earthward moving flux ropes (e.g., Slavin et al., 2003). The main difference in our case is that the magnetic disturbance (TD normals in Table 2) is propagating in a direction not aligned with the velocity of the BBF $\left(\boldsymbol{V}_{\mathrm{HT}}\right)$. Furthermore, our observations are from the outer CPS while BBF type flux ropes observed by Slavin et al. (2003), were typically found in the inner CPS.

The magnetic shear region corresponds to the boundary of the BBF. Sergeev et al. (1996) found that the average thickness of the boundary of depleted flux tubes is $0.3 R_{E}$, or about $2000 \mathrm{~km}$. This value agrees well with our thickness estimates and dimensions from the GS reconstruction.

Field aligned currents are important for the magnetosphere-ionosphere coupling processes. Our estimate of a total current of 0.1 MA is of the same order of magnitude as Shiokawa et al. (1997) found that braking of fast flows at the boundary between the dipolar field lines and the stretched field lines in the tail can contribute. This is $\sim 10 \%$ of the current during large substorms.

Analysis of magnetometer data from the IMAGE chain in northern Scandinavia (Lühr et al., 1998), which is near the Northern Hemisphere footpoint of Cluster for this event, also reveals a westward current of comparable magnitude. A 
more detailed analysis of the ground signatures in connection with this event will be the subject of a future publication.

\section{Conclusions}

We have presented an analysis of a BBF with an Earthward field aligned current at its dawn flank. A Grad-Shafranov reconstruction has been performed of the BBF boundary, and the magnitude of the current has been estimated to be of the order 0.1 MA.

An interesting feature of this event is that the satellites encounter the flow from the dawn side in the outer central plasma sheet. This is identified from the estimated boundary normals which have large $Y$ and $Z$ GSM components and large angles with the propagation direction of the BBF. The curvature radius of a cross section of the flux tube has been estimated to be about $7 R_{E}$. This is in contrast to when the satellites encounter the BBF closer to its front. For such a case, Nakamura et al. (2005) estimated the curvature radius to be $1.5-2 R_{E}$. The boundary of the BBF has been estimated to be about $0.3 R_{E}$ thick.

Inside the boundary there was dipolarization and enhanced $E_{Y}$. The density and temperature are about half and twice of what they are outside. Thus, the BBF has the predicted properties of a depleted flux tube.

Acknowledgements. We would like to thank the International Space Science Institute for giving us the opportunity to meet and exchange ideas in their facilities in Bern. We thank the referees for valuable suggestions for improving the paper.

Topical Editor I. A. Daglis thanks two anonymous referees for their help in evaluating this paper.

\section{References}

Angelopoulos, V., Baumjohann, W., Kennel, C. F., Coroniti, F. V., Kivelson, M. G., Pellat, R., Walker, R. J., Lühr, H., and Paschmann, G.: Bursty bulk flows in the inner central plasma sheet, J. Geophys. Res., 97, 4027-4039, 1992.

Angelopoulos, V., Kennel, C. F., Coroniti, F. V., Pellat, R., Kivelson, M. G., Walker, R. J., Russell, C. T., Baumjohann, W., Feldman, W. C., and Gosling, J. T.: Statistical characteristics of bursty bulk flow events, J. Geophys. Res., 99, 21 257-21 280, 1994.

Balogh, A., Carr, C. M., Acũna, M. H., Dunlop, M. W., Beek, T. J., Brown, P., Fornacon, K.-H., Georgescu, E., Glassmeier, K.-H., Harris, J., Musmann, G., Oddy, T., and Schwingenschuh, K.: The Cluster Magnetic Field Investigation: Overview of in-flight performance and initial results, Ann. Geophys., 19, 1207-1217, 2001, http://www.ann-geophys.net/19/1207/2001/.

Baumjohann, W., Paschmann, G., Sckopke, N., Cattell, C. A., and Carlson, C. W.: Average Ion Moments in the Plasma Sheet Boundary Layer, J. Geophys. Res., 93, 11 507-11 520, 1988.

Baumjohann, W., Paschmann, G., and Cattell, C. A.: Average Plasma Properties in the Central Plasma Sheet, J. Geophys. Res., 94, 6597-6606, 1989.
Baumjohann, W., Paschmann, G., and Lühr, H.: Characteristics of high speed ion flows in the plasma sheet, J. Geophys. Res., 95, 3801-3809, 1990.

Birn, J., Raeder, J., Wang, Y. L., Wolf, R. A., and Hesse, M.: On the propagation of bubbles in the geomagnetic tail, Ann. Geophys., 22, 1773-1786, 2004, http://www.ann-geophys.net/22/1773/2004/.

Cao, J. B., Ma, Y. D., Parks, G., Rème, H., Dandouras, I., Nakamura, R., Zhang, T. L., Zong, Q., Lucek, E., Carr, C. M., Liu, Z. X., and Zhou, G. C.: Joint observations by Cluster satellites of bursty bulk flows in the magnetotail, J. Geophys. Res., 111, A04206, doi:10.1029/2005JA011322, 2006.

Chen, C. X. and Wolf, R. A.: Interpretation of high-speed flows in the plasma sheet, J. Geophys. Res., 98, 21 409-21 419, 1993.

Dunlop, M. W. and Balogh, A.: Magnetopause current as seen by Cluster, Ann. Geophys., 23, 901-907, 2005, http://www.ann-geophys.net/23/901/2005/.

Erickson, G. M. and Wolf, R. A.: Is steady convection possible in the Earth's magnetotail, Geophys. Res. Lett., 7, 897-900, 1980.

Fairfield, D. H., Mukai, T., Brittnacher, M., Reeves, G. D., Kokubun, S., Parks, G. K., Nagai, T., Matsumoto, H., Hashimoto, K., Gurnett, D. A., and Yamamoto, T.: Earthward flow bursts in the inner magnetotail and their relation to auroral brightenings, AKR intensifications, and geosynchronous particle injections and magnetic activity, J. Geophys. Res., 104, 355-370, 1999.

Haaland, S., Sonnerup, B. U. Ö., Dunlop, M. W., Balogh, A., Georgescu, E., Hasegawa, H., Klecker, B., Paschmann, G., PuhlQuinn, P., Rème, H., Vaith, H., and Vaivads, A.: Four-spacecraft determination of magnetopause orientation, motion and thickness: comparison with results from single-spacecraft methods, Ann. Geophys., 22, 1347-1365, 2004, http://www.ann-geophys.net/22/1347/2004/.

Harvey, C. C.: Spatial Gradients and the Volumetric Tensor, in: Analysis Methods for Multi-Spacecraft Data, edited by: Paschmann, G. and Daly, P. W., pp. 307-322, International Space Science Institute, 1998.

Hasegawa, H., Sonnerup, B. U. Ö., Klecker, B., Paschmann, G., Dunlop, M. W., and Rème, H.: Optimal reconstruction of magnetopause structures from Cluster data, Ann. Geophys., 23, 973982, 2005, http://www.ann-geophys.net/23/973/2005/.

Hau, L.-N. and Sonnerup, B. U. Ö.: Two-dimensional coherent structures in the magnetopause: Recovery of static equilibria from single-spacecraft data, J. Geophys. Res., 104, 6899-6917, 1999.

Khrabrov, A. V. and Sonnerup, B. U. Ö.: deHoffmann-Teller Analysis, in: Analysis Methods for Multi-Spacecraft Data, edited by Paschmann, G. and Daly, P. W., pp. 221-248, International Space Science Institute, 1998.

Lühr, H., Aylward, A., Buchert, S. C., Pajunpää, A., Pajunpää, K., Holmboe, T., and Zalewski, S. M.: Weastward moving dynamic substorm features observed with the IMAGE magnetometer network and other ground based instruments, Ann. Geophys, 16, 425-440, 1998.

Mottez, F. and Chanteur, G.: Surface crossing by a group of satellites: A theoretical study, J. Geophys. Res., 99, 13 499-13 507, 1994.

Nakamura, M., Paschmann, G., Baumjohann, W., and Sckopke, 
N.: Ion Distributions and Flows in and Near the Plasma Sheet Boundary Layer, J. Geophys. Res., 97, 1449-1460, 1992.

Nakamura, R., Baumjohann, W., Mouikis, C., Kistler, L. M., Runov, A., Volwerk, M., Asano, Y., Vörös, Z., Zhang, T. L., Klecker, B., Rème, H., and Balogh, A.: Spatial scale of high-speed flows in the plasma sheet observed by Cluster, Geophys. Res. Lett., 31, L09804, doi:10.1029/2004GL019558, 2004.

Nakamura, R., Amm, O., Laakso, H., Draper, N. C., Lester, M., Grocott, A., Klecker, B., McCrea, I. W., Balogh, A., Rème, H., and André, M.: Localized fast flow disturbance observed in the plasma sheet and in the ionosphere, Ann. Geophys., 23, 553566, 2005, http://www.ann-geophys.net/23/553/2005/.

Pontius Jr., D. H. and Wolf, R. A.: Transient flux tubes in the terrestrial magnetosphere, Geophys. Res. Lett., 17, 49-51, 1990.

Raj, A., Phan, T., Lin, R. P., and Angelopoulos, V.: Wind survey of high-speed bulk flows and field-aligned beams in the near-Earth plasma sheet, J. Geophys. Res., 107, 1419, doi:10.1029/2001JA007547, 2002.

Rème, H., Aoustin, C., Bosqued, J. M., Dandouras, I., Lavraud, B., Sauvaud, J. A., Barthe, A., Bouyssou, J., Camus, T., CoeurJoly, O., Cros, A., Cuvilo, J., Ducay, F., Garbarowitz, Y., Medale, J. L., Penou, E., Perrier, H., Romefort, D., Rouzaud, J., Vallat, C., Alcaydé, D., Jacquey, C., Mazelle, C., Uston, C. D., Mobius, E., Kistler, L. M., Crocker, K., Granoff, M., Mouikis, C., Popecki, M., Vosbury, M., Klecker, B., Hovestadt, D., Kucharek, H., Kuenneth, E., Paschmann, G., Scholer, M., Sckopke, N., Seidenschwang, E., Carlson, C. W., Curtis, D. W., Ingraham, C., Lin, R. P., McFadden, J. P., Parks, G. K., Phan, T., Formisano, V., Amata, E., Bavassano-Cattaneo, M. B., Baldetti, P., Bruno, R., Chionchio, G., Lellis, A. D., Marcucci, M. F., Pallocchia, G., Korth, A., Daly, P. W., Graeve, B., Rosenbauer, H., Vasyliunas, V., McCarthy, M., Wilber, M., Eliasson, L., Lundin, R., Olsen, S., Shelley, E. G., Fuselier, S., Ghielmetti, A. G., Lennartsson, W., Escoubet, C. P., Balsiger, H., Friedel, R., Cao, J.-B., Kovrazhkin, R. A., Papamastorakis, I., Pellat, R., Scudder, J., and Sonnerup, B.: First multispacecraft ion measurements in and near the Earth's magnetosphere with the identical Cluster ion spectrometry (CIS) experiment, Ann. Geophys., 19, 1303-1354, 2001, http://www.ann-geophys.net/19/1303/2001/.
Robert, P., Dunlop, M. W., Roux, A., and Chanteur, G.: Accuracy of Current Density Determination, in: Analysis Methods for MultiSpacecraft Data, edited by Paschmann, G. and Daly, P. W., pp. 395-418, International Space Science Institute, 1998a.

Robert, P., Roux, A., Harvey, C. C., Dunlop, M. W., Daly, P. W., and Glassmeier, K.-H.: Tetrahedron Geometric Factors, in: Analysis Methods for Multi-Spacecraft Data, edited by: Paschmann, G. and Daly, P. W., pp. 323-348, International Space Science Institute, $1998 \mathrm{~b}$.

Sergeev, V. A., Angelopoulos, V., Gosling, J. T., Cattell, C. A., and Russel, C. T.: Detection of localized, plasma-depleted flux tubes or bubbles in the midtail plasma sheet, J. Geophys. Res., 101, 10817-10 826, 1996.

Shiokawa, K., Baumjohann, W., and Haerendel, G.: Braking of high-speed flows in the near-Earth tail, Geophys. Res. Lett., 24, 1179-1182, 1997.

Siscoe, G. L., Davis Jr., L., Coleman Jr., P. J., Smith, E. J., and Jones, D. E.: Power spectra and discontinuities of the interplanetary magnetic field: Mariner 4, J. Geophys. Res., 73, 61-82, 1968.

Slavin, J. A., Lepping, R. P., Gjerloev, J., Fairfield, D. H., Hesse, M., Owen, C. J., Moldwin, M. B., Nagai, T., Ieda, A., and Mukai, T.: Geotail observations of magnetic flux ropes in the plasma sheet, J. Geophys. Res., 108, 1015, doi:10.1029/2002JA009557, 2003.

Sonnerup, B. U. Ö., Haaland, S., Paschmann, G., Dunlop, M. W., Rème, H., and Balogh, A.: Orientation and motion of a plasma discontinuity from single-spacecraft measurements: Generic residue analysis of Cluster data, J. Geophys. Res., 111, A05203, doi:10.1029/2005JA011538, 2006. 